\title{
On the Probability of Detection Ability in Observing Dynamic Environmental Phenomena using Wireless Sensor Networks
}

\author{
Omar Fouad Mohammed \\ Faculty of information and \\ communication technology, \\ Universiti Teknikal Malaysia Melaka \\ (UTeM), \\ Melaka, Malaysia
}

\author{
Burairah Hussin \\ Faculty of information and \\ communication technology, \\ Universiti Teknikal Malaysia Melaka \\ (UTeM), \\ Melaka, Malaysia
}

\author{
Abd Samad Hasan Basari \\ Faculty of information and \\ communication technology, \\ Universiti Teknikal Malaysia Melaka \\ (UTeM), \\ Melaka, Malaysia
}

\begin{abstract}
Wireless Sensor Network (WSN) is being utilised for several purposes in military and civil domains, including surveillance, monitoring, and management, where networked sensors monitor and detect an event of interest and report to the concerned party through the WSN or the Internet infrastructure. Due to the characteristics of WSN, there are many fundamental technical challenges including the node deployment, event localization, and event tracking, among which, the probability of the event observability has a crucial role. The observability is defined as the capability of observing an evolving event in the monitoring area. The probability of detection ability in observing an event depends on the parameters of the detection function, which in turn rely on the sensor technology and the nature of the surrounding environment. This paper addresses the observation of an event using WSN and how accurately the event is observed in the monitoring area. It presents a practical solution for event observability after formulizing and establishing the complexity of observability issue and tackling its relation and impact on node deployment and event localization. Hence, a feasible event observation model has been proposed and validated in this paper. The numerical results of the experimental evaluation have confirmed that an accurate detection of an occurring event can be achieved by the proposed model.
\end{abstract}

Keywords-Wireless Sensor Network (WSN); Environmental Monitoring; Event Detection; Event Localization; Sensing Modelling

\section{INTRODUCTION}

The rapid development in Micro-Electro-Mechanical Systems (MEMs) and the computation and communication technologies paved the way for the advances in Wireless Sensor Networks (WSNs). Sensors have the ability to operate independently, perform intended sensing tasks, process the sensed data, and report to a particular interested remote station $[3,14,15]$. Besides these advantages, the low cost and reliability of sensors have facilitated the establishment of numerous applications for various monitoring and management purposes including environment, transportation, human activity, detection and target tracking, underground mining and pipeline (such as water, oil, gas), healthcare , precision agriculture, industrial and supply chain [9].
Hence, WSN is being deployed for various monitoring and management operations in military and civil domains, where a specific event is observed and reported to the interested entity via the WSN or the Internet infrastructure [19, 18]. Accordingly, there have been a lot of research works on the development and practice of WSNs including architectures, operating systems, and applications. Nonetheless, the features of WSNs impose some fundamental technical challenges including the deployment of the sensor nodes, localization of the occurring event, and tracking of the event evolution, among which the probability of the event observability is an essential factor.

The observability of the event is defined as the capability of detecting an event (such as an environmental phenomenon) occurred in the monitoring area. The observability is related to the probability of sensing an event by sensors, where high observability indicates that there is a higher possibility that an event can be detected by the nearby sensors. It can be theoretically computed as the integral of the detection function considering the distance between the detecting sensors and the trajectory of the event $e$ that is progressing from point $p_{i}$ to another point $p_{i+1}$. The probability of detection ability in observing an event depends on the detection function parameters, which in turn rely on the technology of the sensor and the nature of the surrounding environment. The wellknown detection model used in seismic sensors, which are used to measure seismic vibrations by converting ground motion into a measurable electronic signal, takes the form of $\lambda * d\left(s_{i}, p_{i}\right)^{-\beta}$, where $\lambda$ and $\beta$ are sensor technologies and environment parameters respectively, and $d\left(s_{i}, p_{i}\right)$ is the distance from sensor $i$ to an event at point $i[8,4,6,13]$.

This paper focuses on how accurately an occurring event can be observed in the monitoring area. The objectives of the research work presented in this paper are to come up with a practical solution for event observability once formulizing and establishing the complexity of observability problem, and to tackle its relation and impact on sensors deployment and event localization. The rest of the paper is outlined as: Section 2 presents some of the related research works. The common feasible sensing models and the proposed event 
observation model that is based on probabilistic concept along with several effective observability measures presented in Section 3. Section 4 presents the experimental evaluation for validating the proposed event observation model. Finally, Section 5 presents the conclusion of the research work presented here and suggests some potential future works.

\section{RELATED WORK}

There are several research works, such as the ones presented in $[1,2,5,7,10,11,12,13,16,17,20,21,22,23$, $24,25]$, which have been focusing on the detection coverage and the observability path of a targeted event. Among the main objectives of these research works, a quantitative measure that reflects how accurate an event can be detected by the sensors in the monitoring area was of concern, while considering the location of the event development paths (that are highly likely can be detected by the nearby sensors), in addition to the effect of the sensor deployment on the detection of a target, while increasing the observability of the least observed path in the monitoring area. These research works presented the advantage of the probabilistic detection over the deterministic one. With a probabilistic detection model, the sensor $s_{i}$ is capable of detecting an event located at point $p_{i}$ with probability $P\left(D\left(d\left(s_{i}, p_{i}\right)\right)\right)$ which is defined as a decreasing function; that is $0 \leq P\left(D\left(d\left(s_{i}, p_{i}\right)\right) \leq 1\right.$. Moreover, with this model, the joint detection probability of a location point $p$ (which is covered by some sensors) can be used to quantify the coverage of $p$. When the joint detection probability is greater than a predefined threshold, then $p$ is definitely covered.

Some of the research works available in the literature had focused on a deterministic coverage of a location point, while other works had aimed at a probabilistic coverage. However, the coverage of whole monitoring area was not addressed properly and no solutions were introduced to ensure the coverage of the entire area. Moreover, most of the researches on detection coverage supposed a perfect disc detection model (also known as the binary detection model) where the sensing range is fixed. With such model, an event would definitely be detected in the monitoring area if it occurs within the sensing range $\operatorname{sen}_{r}$ of a sensor $i$; that is, the event is detected if its distance $d$ to the closest sensor $i$ is less than $i$ 's sensing range $\operatorname{sen}_{r}$. Nevertheless, this is considered as a rough approximation since the event detection characteristically relies on various variables and techniques used to confirm the detection accuracy. Thus, for a superior approximation, in every sensor, a probabilistic detection model with respect to the Euclidean distance between a sensing node and the event located at point $p$ should be taken into account.

While these works were directed to coverage-related algorithm design, and that their network coverage formulations consider the distance between the nearest sensors to the event, for the concept presented in this paper, the probability of the observability in the monitoring area is characterised and calculated as an integral of several sensing measures and asymptotic behaviours.

\section{DETECTION MODELS AND EFFECTIVE OBSERVABILITY MEASURES}

With consideration of the closest distance between the sensor and the event, most of the current proposed works use a fixed detection radius, within which a sensing node $i$ would certainly detects the event once triggered in the monitoring area. The majority of event detection applications in WSNs require that nodes should be activated probabilistically to sense the surrounding area. A linear function that is inversely proportional to the detection accuracy can help in demonstrating the distance from the node to a particular point where the event has happened. Hence, the detection probability $P\left(D_{i}\right)$ of node $i$ can be computed as follows:

$$
\begin{aligned}
& P\left(D_{i}\right)=1 /\left(1+\lambda * d\left(s_{i}, p_{i}\right)\right)^{\beta} \\
& P\left(D_{i}\right)=1+\lambda * d\left(s_{i}, p_{i}\right)^{-\beta}
\end{aligned}
$$

where, $d\left(s_{i}, p_{i}\right)$ is the measurable distance from a sensing node $i$ to an event at a point $p$. $\lambda$ and $\beta$ are sensor technologies and environment parameters respectively, where $\lambda$ is a tuning parameter and that $\beta$ ranges between 1 and 4 according to the surrounding environment.

Also, an exponential function of the distance can define the detection probability inversely as follows:

$$
P\left(D_{i}\right)=e^{-\left(\lambda * d\left(s_{i}, p_{i}\right)\right)}
$$

Furthermore, the detection probability model can be presented as combination of linear and exponential functions constrained by two limiting thresholds ( $\min$, max), such that:

$$
P\left(D_{i}\right)= \begin{cases}1, & d\left(s_{i}, p_{i}\right)<\min \\ 0, & d\left(s_{i}, p_{i}\right)>\max \\ \lambda e^{-\left(\beta * d\left(s_{i}, p_{i}\right)\right)}, & \min <d\left(s_{i}, p_{i}\right)<\max \end{cases}
$$

While the detection probability model introduced in Equation (4) is reasonable in comparison to the previous ones, though it has suitability constraint. To provide more practical detection model, the probability to detect an occurring event can be presented as follows:

$$
P\left(D_{i}\right)=\lambda \alpha^{-\left(\beta * d\left(s_{i}, p_{i}\right)\right)^{\tau}}
$$

where,

$\lambda$ presents the accuracy of the observability; it defines the maximum probability of that an event is definitely detected by the sensing node $i$, such that $0<\lambda \leq 1$; i.e. $\lambda=1$ in case $d\left(s_{i}, p_{i}\right)=0$.

$\alpha$ and $\beta$ presents the vertical and the horizontal locales respectively, where $\alpha>1$ and $\beta>0$. A formulation of probability distribution can be made with respect to a reference point $q$ which can be characterised by $\left(d_{q}\left(s_{i}, q\right), P_{q}\left(D_{i}\right)\right)$. This implies that when an event occurs at $d_{q}\left(s_{i}, q\right)$ distance away from the sensing node $i$, the probability of detecting the event is $P_{q}\left(D_{i}\right)$. Thus, when $\beta d_{q}\left(s_{i}, q\right)=1, P_{q}\left(D_{i}\right)=\lambda \alpha^{-1}$, which 
allows determining a reference point $\left(d_{q}\left(s_{i}, q\right), P_{q}\left(D_{i}\right)\right)$ by defining the parameters $\alpha$ and $\beta$ as follows:

$$
\begin{aligned}
& \alpha=\lambda *\left(P_{q}\left(D_{i}\right)\right)^{-1} \\
& \beta=d_{q}\left(s_{i}, q\right)^{-1}
\end{aligned}
$$

$\tau$, $(\tau>0)$, represents decreasing tendency of the detection probability $\lambda$ to 0 , as for $d\left(s_{i}, p_{i}\right)$. When there is a need to specify that at a particular distance $d^{\prime}\left(s_{i}, p_{i}\right)$, the detection probability is $P^{\prime}\left(D_{i}\right)$, then $\tau$ must be as follows:

$$
\tau=\log d_{\beta * d^{\prime}\left(s_{i}, p_{i}\right)} \log _{\beta}\left(\frac{\lambda}{P^{\prime}\left(D_{i}\right)}\right)
$$

provided that the conditions $d^{\prime}\left(s_{i}, p_{i}\right)>d_{q}\left(s_{i}, q_{r}\right)$, and $P^{\prime}\left(D_{i}\right)<P_{q}\left(D_{i}\right)$, or vice versa, should be maintained.

As highlighted previously, with a fixed radius-based detection model, a node would surely detect any event occurring within its sensing range $\left(\operatorname{sen}_{r}\right)$; thus, in such scenario, the detection probability would be as follows:

$$
P\left(D_{i}\right)= \begin{cases}1, & d\left(s_{i}, p_{i}\right)<\operatorname{sen}_{r} \\ 0, & \text { otherwise }\end{cases}
$$

Sensing Area $(S A)$ is the network coverage at any given point $i\left(p_{i}\right)$, which is interpreted as the probability with which a sensing node can detect an event at $p_{i}$. Hence, it can be computed as follows:

$$
S A\left(p_{i}\right)=1-\Pi\left(1-P\left(D_{i}\right)\right)
$$

where, $S A\left(p_{i}\right)$ is the coverage at $\left(p_{i}\right)$, and $P\left(D_{i}\right)$ is the detection probability of the sensing node $i$ at $p_{i}$ of the monitoring area.

The coverage of the sensing area $C\left(S A, P_{i}\right)$ indicates that the sensing area $S A$ is the overall achieved detections from nodes in the monitoring area at $p_{i}$.

Therefore, when there are $n$ sensor nodes in the network, the coverage of the sensing area at $p_{i}$ can be as follows:

$$
C\left(S A, p_{i}\right)=\sum_{1}^{n}\left(s_{i}, p_{i}\right)
$$

The proposed model can be useful for detecting events that occur in indoor and outdoor environments such as intruders, fire outbreaks, gas leak, and so on. Such events are considered dynamic and in order to be accurately detected the observation and localization must be performed properly.

\section{EXPERIMENTAL EVALUATION}

In order to validate the proposed probabilistic detection model and its ability to observe and localise a dynamic environmental phenomena a comprehensive experimental evaluation has been conducted using simulation. This section presents the scenario and settings used in the experimental evaluation followed by the discussion on the results gained from the simulation experiments.

\section{A. Experiments Settings}

A network of 1000 of wireless sensor nodes has been simulated using Network Simulator 2 (ns-2) running on a computing station with a CentOS version of Linux. The sensor nodes were deployed randomly over an area of $250 \times$ $250 \mathrm{~m}^{2}$ to monitor the surrounding environment. Hence, the density of sensor nodes is 0.016 per $\mathrm{m}^{2}$. Each sensor node has a sensing range $\left(\right.$ sen $\left._{r}\right)$ of $30 \mathrm{~m}$ and a transmission range $\left(T_{x}\right)$ of $120 \mathrm{~m}$ which enables a direct transmission of the detection data to a base station located at the centre of the sensing area. The settings of associated parameters of the sensing model were as follows: $\lambda=1$ (100\% observation), $\alpha=2, \beta=0.1$ (50\% observation of event occurred at $30 \mathrm{~m})$, and $\tau=4$. The evaluation investigates the effect of every parameter on the subject of density that varies between 0.01 and 0.05 , and compared to the fixed detection radius sensing model where $\operatorname{sen}_{r}=30 \mathrm{~m}$.

\section{B. Evaluation Results}

The results presented in this section are regarding the effect of the observability accuracy parameter $\lambda$, the vertical locale $\alpha$, the horizontal locale $\beta$, and the decreasing tendency of the probability of the observability accuracy $\tau$ parameters.

\section{1) Impact of the Observability Accuracy $\lambda$}

The observation accuracy of an event can be well demonstrated by this parameter. As there is no guarantee that a sensor node can always observe an event once it happens. This is because of some limitations associated with the sensor measurement and the nature of the event. The observability accuracy parameter is examined with different values where $\lambda=0.6, \lambda=0.7, \lambda=0.8, \lambda=0.9$, and $\lambda=1.0$, respectively; and in comparison with the fixed detection radius sen $n_{r}$ of $30 \mathrm{~m}$. Thus, when $d\left(s_{i}, p_{i}\right)=0$, the probability of the detection accuracy $P\left(D_{i}\right)$ to observe the event is $0.6,0.7,0.8,0.9$, and 1.0, respectively. Figure 1 illustrates the effect of the observability accuracy considering the node density and the average detection observations of the event at a given point $i$ in the monitoring area. The results imply that in case $\lambda=1$, the average number of detections is closely equivalent to the fixed radius case at certain values of node density of 0.02 and 0.03; while it is higher as the node density increases. The results confirm that better event detection accuracy is provided by the proposed detection model; hence, reducing the possibilities of having false alarms of the event. In addition, for various settings of $\lambda$, the number of event detections increases as the node density increases, confirming the occurrence of the event that is observed by several sensor nodes. 


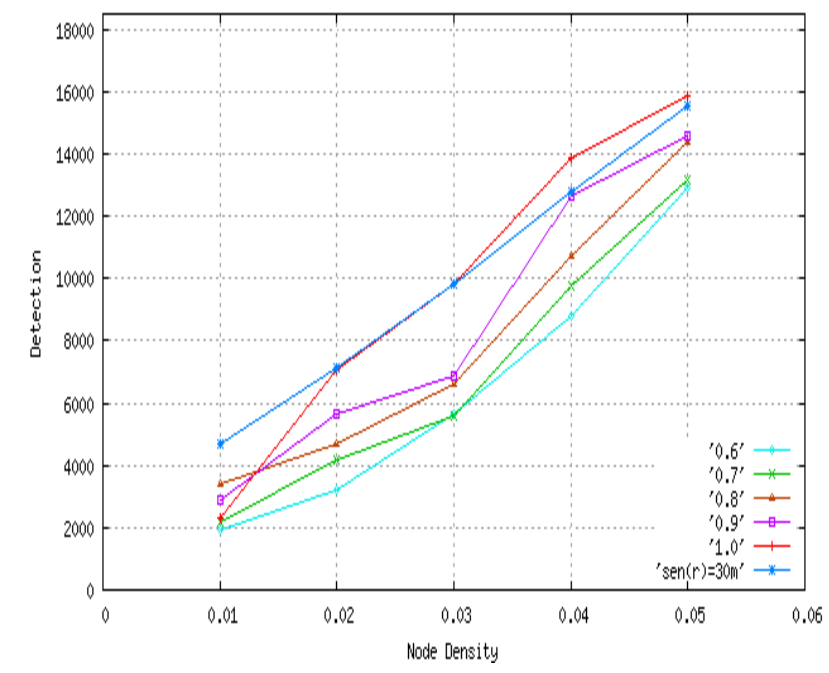

Fig. 1. Impact of the observability accuracy $\lambda$, where $\lambda=0.6, \lambda=0.7$, $\lambda=0.8, \lambda=0.9, \lambda=1.0$

2) Impact of the Vertical Locale $\alpha$

This parameter defines the probability $P_{q}\left(D_{i}\right)$ of observing an event occurring at a specific reference point $q$; that is, $d_{q}\left(s_{i}, q\right)$. It has been examined with different values: $\alpha=1 / 0.6, \alpha=1 / 0.7, \alpha=1 / 0.8$, and $1 / 0.9$ respectively; as presented in Figure 2. This implies that, with respect to the point located at $d_{q}\left(s_{i}, q\right)$, the resulted probability values are $0.6,0.7,0.8$, and 0.9 , respectively. The detection probability for the reference point is increasing when decreasing the value of $\alpha$. This implies that the detection probability is higher when the event happens at $d_{q}\left(s_{i}, q\right)$ away from the sensor node(s). This is confirmed by the results behaviour presented in Figure 2 . As it can be seen in the figure, when $\alpha=1 / 0.7$, the results are close to that of the fixed detection radius when the node densities are 0.03 and 0.04, implying that such settings are identical to the fixed detection radius of $30 \mathrm{~m}$.

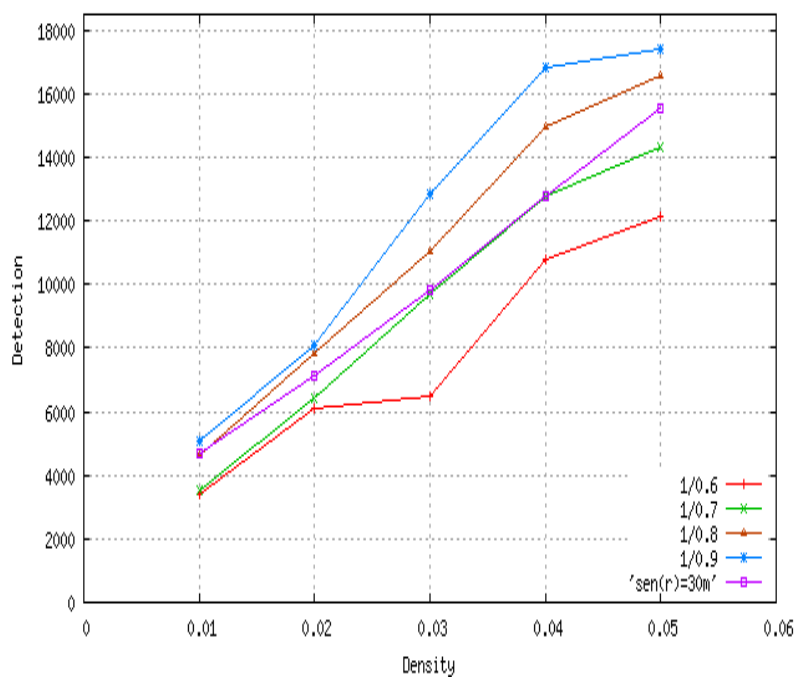

Fig. 2. Impact of the vertical locale $\alpha$, where $\alpha=1 / 0.6, \alpha=1 / 0.7$, $\alpha=1 / 0.8, \alpha=1 / 0.9$
3) Impact of the Horizontal Locale $\beta$

This parameter specifies the distance between the reference point $q$ and the sensor node $i$ detecting the event (i.e. $\left.d_{q}\left(s_{i}, q\right)\right)$. The parameter has been examined with various settings where $\beta=1 / 35, \beta=1 / 30, \beta=1 / 25$, which means that the reference point $q$ is located at $35 \mathrm{~m}, 30 \mathrm{~m}$, and $25 \mathrm{~m}$ away from the sensor node $i$, respectively. Figure 3 shows that the distance $d_{q}\left(s_{i}, q\right)$ becomes higher as $\beta$ value decreases, which implies that a sensor placed at a greater distance would have a higher probability to detect the occurring event. Compared to the rest of the settings, the results in the figure demonstrate that the closest statistical results to the fixed detection radius appear when $\beta=1 / 30$ (i.e $d_{q}\left(s_{i}, q\right)=30 \mathrm{~m}$ ).

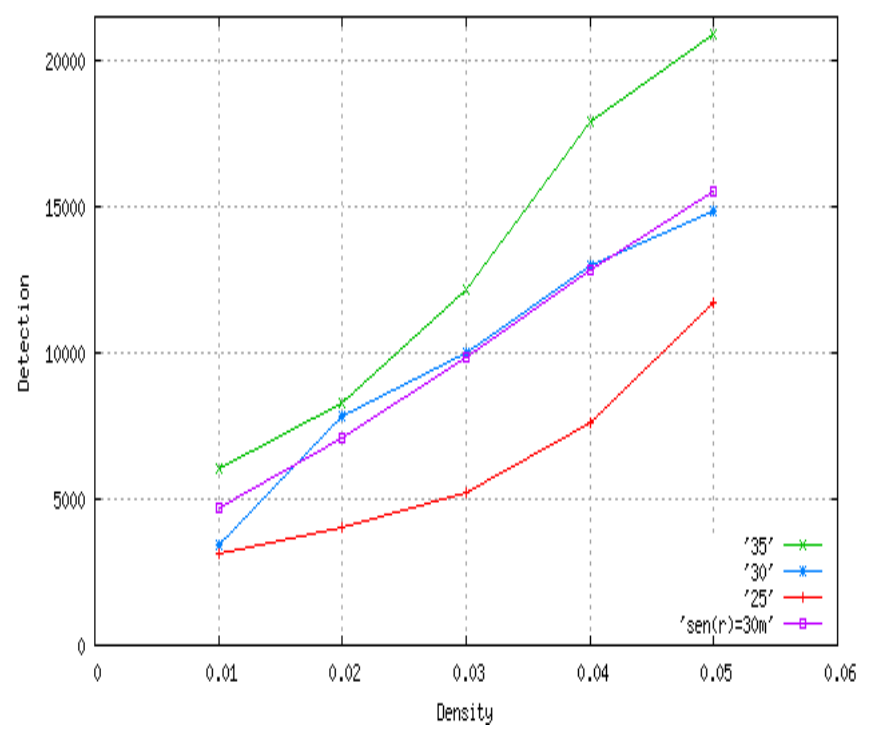

Fig. 3. Impact of the horizontal locale $\beta$, where $d_{q}\left(s_{i}, q\right)=35 \mathrm{~m}, 30 \mathrm{~m}, 25 \mathrm{~m}$

4) Impact of the probability decreasing tendency $\tau$

This parameter defines how steep the slop of the probability of the observation decreases. It has been examined with different values where, $\tau=8, \tau=6$, and $\tau=4$, respectively. Increasing $\tau$ causes a sharp decrease in the probability of the observation accuracy. This implies that, with higher settings of $\tau$, the proposed detection model is driven to follow the course of the fixed detection radius model. This is verified by the results presented in Figure 4 where the results are almost comparable when $\tau=8$ and $d_{q}\left(s_{i}, q\right)=\operatorname{sen}_{r}=30 \mathrm{~m}$ for various node densities. Thus, the lower value of $\tau$ is the lesser the steep of the observation probability. 


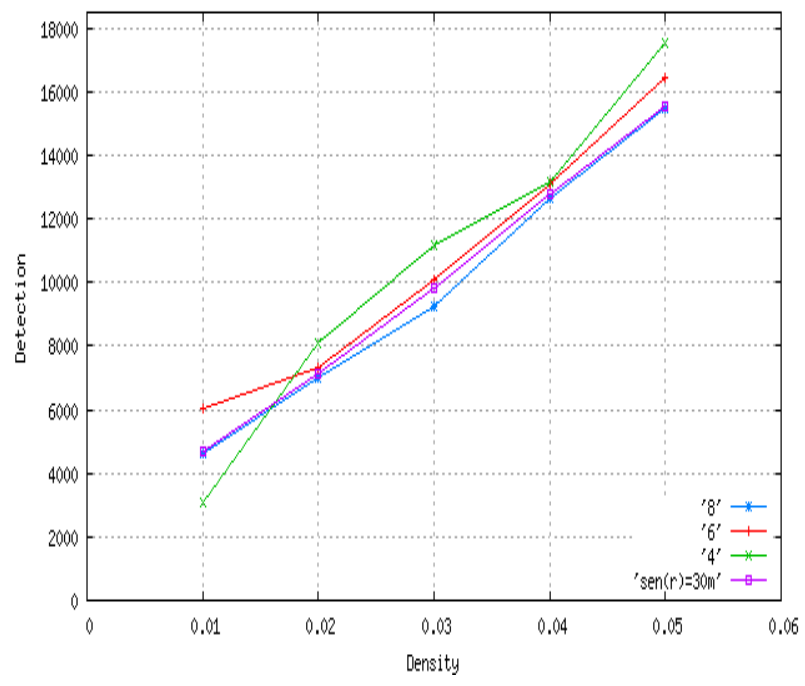

Fig. 4. Impact of the decreasing tendency $\tau$, where $\tau=8, \tau=6, \tau=4$

\section{CONCLUSION AND FUTURE WORK}

In this paper, an event observation model that is based on probabilistic concept has been presented. Also, some effective observability measures with the use of Wireless Sensor Network (WSN) for sensing and locating of an abnormal event have been introduced. Four parameters have adapted to reflect various environment scenarios, which are: observability accuracy $\lambda$, the vertical locale $\alpha$, the horizontal locale $\beta$, and the decreasing tendency $\tau$ of the detection probability. The ability of the proposed model in observing a dynamic environmental phenomenon is well investigated with various settings for the above-mentioned parameters, in terms of the average number of event detections with respect to different node densities, and in comparison with the fixed detection radius model. The experimental evaluation results confirmed that the proposed event observation model provides a proper analytical description of the detectability of an event and it can be utilised for various monitoring applications where sensing coverage is of concern. In the future work, the direction of the research is going towards exploring the potential course of the event evolution to provide an accurate estimation and tracking of the development of the event.

\section{ACKNOWLEDGMENT}

This work was supported by Faculty of Information and Communication Technology, Universiti Teknikal Malaysia Melaka (UTeM).

\section{REFERENCES}

[1] Nadeem Ahmed, Salil S Kanhere, and Sanjay Jha, "Probabilistic coverage in wireless sensor networks," Proceedings of The IEEE Conference on Local Computer Networks 30th Anniversary, 2005, pp. 672-681.

[2] V. Akbarzadeh, C. Gagne, M. Parizeau, M. Argany, and M. A. Mostafavi, "Probabilistic sensing model for sensor placement optimization based on line-of-sight coverage," IEEE Transactions on Instrumentation and Measurement, vol. 62, no. 2, pp. 293-303, 2013.

[3] M. Akter, M.O. Rahman, M.N. Islam, and M.A. Habib, "Incremental clustering-based object tracking in wireless sensor networks," Proceedings of the International Conference on Networking Systems and Security, 2015, pp. 1-6.
[4] Tatiana Bokareva, Wen Hu, Salil Kanhere, Branko Ristic, Neil Gordon, Travis Bessell, Mark Rutten, and Sanjay Jha, "Wireless sensor networks for battlefield surveillance," Proceedings of the land warfare conference, 2006, pp. 1-8.

[5] M. Cardei and J. Wu, "Coverage problems in wireless ad hoc sensor networks," in Handbook of Sensor Networks, FL, Boca Raton:CRC Press, 2004.

[6] Jiming Chen, Junkun Li, Shibo He, Youxian Sun, and Hsiao-Hwa Chen, "Energy-efficient coverage based on probabilistic sensing model in wireless sensor networks," IEEE communications letters, vol. 14, no. 9, pp. 833-835, 2010.

[7] Jiming Chen, Junkun Li, and Ten H Lai, "Energy-efficient intrusion detection with a barrier of probabilistic sensors: Global and local," IEEE Transactions on Wireless Communications, vol. 12, no. 9, pp. 47424755, 2013.

[8] Thomas Clouqueur, Veradej Phipatanasuphorn, Parameswaran Ramanathan, and Kewal K Saluja, "Sensor deployment strategy for target detection," Proceedings of the 1st ACM international workshop on Wireless sensor networks and applications, 2002, pp. 42-48.

[9] Waltenegus Dargie and Christian Poellabauer, "Fundamentals of wireless sensor networks theory and practice," John Wiley \& Sons, 2010.

[10] X. Fan, Q. Chen, Z. Che, and X. Hao, "Energy-efficient probabilistic barrier construction in directional sensor networks," IEEE Sensors Journal, vol. 17, no. 3, pp. 897-908, 2017.

[11] Shibo He, Jiming Chen, Xu Li, Xuemin Shen, and Youxian Sun, "Costeffective barrier coverage by mobile sensor networks," Proceedings of the IEEE INFOCOM, 2012, pp. 819-827.

[12] Mohamed Hefeeda and Hossein Ahmadi, "Energy-efficient protocol for deterministic and probabilistic coverage in sensor networks," IEEE Transactions on Parallel and Distributed Systems, vol. 21, no. 5, pp. 579-593, 2010.

[13] Ashraf Hossain and Rashmita Mishra, "Sensing and link model for wireless sensor network: Coverage and connectivity analysis," arXiv preprint arXiv:1406.1275, 2014.

[14] Xing Hu, Shiqiang Hu, Lingkun Luo, and Guoxiang Li, "Abnormal event detection in crowded scenes via bag-of-atomic-events-based topic model," Turkish Journal of Electrical Engineering \& Computer Sciences, vol. 24, no. 4, pp. 2638-2653, 2016.

[15] Agaji Iorshase and Shangbum F Caleb, "A neural based experimental fire-outbreak detection system for urban centres," Journal of Software Engineering and Applications, vol. 9, no. 3, pp. 71-79, 2016.

[16] Gaurav S Kasbekar, Yigal Bejerano, and Saswati Sarkar, "Lifetime and coverage guarantees through distributed coordinate-free sensor activation," IEEE/ACM transactions on networking, vol. 19, no. 2, pp. 470-483, 2011.

[17] Benyuan Liu and Don Towsley, "A study of the coverage of large-scale sensor networks," Proceedings of the IEEE International Conference on Mobile Ad-hoc and Sensor Systems, 2004, pp. 475-483.

[18] Xiaoxi Liu, Ruiying Li, and Ning Huang, "A sensor deployment optimization model of the wireless sensor networks under retransmission," Proceedings of the 4th Annual IEEE International Conference on Cyber Technology in Automation, Control, and Intelligent Systems, 2014, pp. 413-418.

[19] Markus Quaritsch, Karin Kruggl, Daniel Wischounig-Strucl, Subhabrata Bhattacharya, Mubarak Shah, and Bernhard Rinner, "Networked UAVs as aerial sensor network for disaster management applications," e \& i Elektrotechnik und Informationstechnik, vol. 127, no. 3, pp. 56-63, 2010.

[20] Y. Tian, X. Lin, G. You, and H. Zhang, "A node schedule method for multi-coverage probability based on probabilistic coverage in wsns," Proceedings of the Chinese Control and Decision Conference, 2016, pp. 2385-2389.

[21] H. L. Wang and W. H. Chung, "The generalized k-coverage under prob abilistic sensing model in sensor networks," Proceedings of the IEEE Wireless Communications and Networking Conference, 2012, pp. 17371742 . 
[22] Guoliang Xing, Chenyang Lu, Robert Pless, and Joseph A O'Sullivan, "Co-grid: an efficient coverage maintenance protocol for distributed sensor net works," In Proceedings of the 3rd international symposium on Information processing in sensor networks, ACM, 2004, pp. 414-423.

[23] Guoliang Xing, Rui Tan, Benyuan Liu, Jianping Wang, Xiaohua Jia, and Chih-Wei Yi, "Data fusion improves the coverage of wireless sensor networks," In Proceedings of the 15th annual international conference on Mobile computing and networking, ACM, 2009, pp. 157-168.
[24] Q. Yang, S. He, J. Li, J. Chen, and Y. Sun, "Energy-efficient probabilistic area coverage in wireless sensor networks," IEEE Transactions on Vehicular Technology, vol. 64, no. 1, pp. 367-377, 2015.

[25] Yi Zou and Krishnendu Chakrabarty, "Sensor deployment and target localization in distributed sensor networks," ACM Transactions on Embedded Computing Systems, vol. 3, no. 1, pp. 61-91, 2004. 